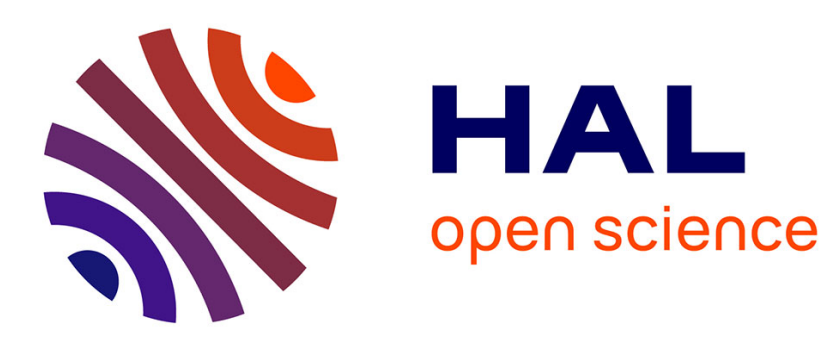

\title{
Correlation effects and Energetics of point defects in uranium dioxide: a first principle investigation
}

Alain Pasturel, Florence Gupta, Guillaume Brillant

\section{To cite this version:}

Alain Pasturel, Florence Gupta, Guillaume Brillant. Correlation effects and Energetics of point defects in uranium dioxide: a first principle investigation. Philosophical Magazine, 2008, 87 (17), pp.25612569. 10.1080/14786430701235814 . hal-00513819

\section{HAL Id: hal-00513819 https://hal.science/hal-00513819}

Submitted on 1 Sep 2010

HAL is a multi-disciplinary open access archive for the deposit and dissemination of scientific research documents, whether they are published or not. The documents may come from teaching and research institutions in France or abroad, or from public or private research centers.
L'archive ouverte pluridisciplinaire HAL, est destinée au dépôt et à la diffusion de documents scientifiques de niveau recherche, publiés ou non, émanant des établissements d'enseignement et de recherche français ou étrangers, des laboratoires publics ou privés. 


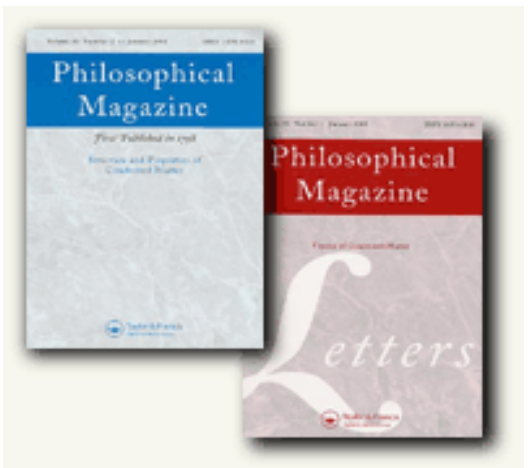

\section{Correlation effects and Energetics of point defects in uranium dioxide: a first principle investigation}

\begin{tabular}{|r|l|}
\hline Journal: & Philosophical Magazine \& Philosophical Magazine Letters \\
\hline Manuscript ID: & TPHM-06-Nov-0451.R1 \\
\hline Journal Selection: & Philosophical Magazine \\
\hline Author: & 22-Dec-2006 \\
\hline Complete List of Authors: & $\begin{array}{l}\text { Pasturel, Alain; Universite de Grenoble, Laboratoire de Physique et } \\
\text { Modelisation des Milieux Condenses } \\
\text { Gupta, Florence; Institut de Radioprotection et de Sûreté Nucléaire } \\
\text { Brillant, Guillaume; Institut de Radioprotection et de Sûreté } \\
\text { Nucléaire }\end{array}$ \\
\hline Keywords: & density-functional methods, point defects \\
\hline Keywords (user supplied): & \\
\hline
\end{tabular}

\section{S ScholarONE \\ Manuscript Central}




\title{
Correlation effects and Energetics of point defects in uranium dioxide: a first principle investigation
}

\author{
F. Gupta and G. Brillant \\ Institut de Radioprotection et de Sûreté Nucléaire, DPAM, SEMIC, LEPF, Centre d'Etudes \\ Nucléaires de cadarache, BP 313115 Saint Paul Lez Durance Cedex, France
}

A. Pasturel

Laboratoire de Physique et Modélisation des Milieux Condensés (LPMMC), Maison des magistères - CNRS, BP166, 38042 Grenoble Cedex09, France

\begin{abstract}
:
Density functional (DFT) calculations have been used to investigate the stability of point defects in uranium dioxide. Correlation effects are taken into account within the DFT+U approach as implemented in the Vienna Ab initio Simulation Package (VASP). More particularly the formation energies of both intrinsic and extrinsic point defects, i.e. vacancies, interstitials, Frenkel pairs and Schottky trio defects are calculated. Our results are compared with available experimental data and are also discussed in relation to previous calculations based on conventional functionals such as the local spin-density approximation and generalized gradient approximations.
\end{abstract}

Corresponding author: pasturel@grenoble.cnrs.fr 


\section{INTRODUCTION:}

Uranium dioxide $\left(\mathrm{UO}_{2}\right)$ is of long-standing interest to material scientists due to the diversity of the fundamental problems associated with the material and by the important technological problems arising from its use as a reactor fuel cell. $\mathrm{UO}_{2}$ is known to be an antiferromagnetic insulator [1,2] with an optical gap of roughly $2 \mathrm{eV}$. However it is also known that its transport properties, both atomic and electronic, as well as thermodynamic properties, are greatly influenced by the deviations from stoichiometric composition, which are caused by the occurrence of point defects. More particularly, such defects can modify the crystal structure, cause a swelling of the solid and also may constitute traps for fission gases or helium. Then they have a direct impact on thermomechanical, structural an kinetic properties of the material, especially under irradiation.

In view of the importance of $\mathrm{UO}_{2}$ and of the difficulty that is found in experimental studies (particularly under the extreme conditions of the nuclear industry) there is a need for reliable theoretical calculations of defect formation and migration energies. The first theoretical studies of point defect formation energies were based on interionic empirical potentials using the Mott-Littleton methodology [3-5]. However the calculated formation energies of uranium Frankel pair and that of Schottky trios present an important discrepancy (about a factor of 2) with experimental values deduced from diffusion coefficient measurements [6]. Such an overestimation is essentially due to the use of formal ions charges to describe cohesive properties of partially covalent oxides. More recently, ab initio calculations, based on the density functional theory (DFT) were used to calculate formation energies of point defects in $\mathrm{UO}_{2}$. In these calculations, the major problem is that both the local density approximation (LDA) and the generalized gradient approximations (GGA) give a qualitatively incorrect description of the strongly correlated $5 \mathrm{f}$ states of $\mathrm{U}$, leading to metallic instead of insulating behaviour of $\mathrm{UO}_{2}$ [7-9]. To correct these well known failures of the conventional DFT based calculations, more sophisticated functionals were introduced recently to describe the insulating behaviour of $\mathrm{UO}_{2}$. For instance, calculations using hybrid DFT functional $[10,11]$ give results in very good agreement with experimental photoemission data. The self-interaction correction is also known to correct this kind of problems [12]. Dudarev et al. [13] showed that DFT+U method yields the correct insulating ground state. Very recently, 


\section{METHOD :}

Total energy calculations were performed with the projector-augmented-wave method [15] as implemented in the Vienna Ab initio Simulation package [16]. The effects due to the localization of the $\mathrm{f}$ electrons of uranium in $\mathrm{UO}_{2}$ are taken into account with the GGA+U approximation as recently implemented in VASP $[17,18]$. In the so-called GGA+U approximation, the spin-polarized GGA potential [19] is supplied by a Hubbard-like term to account for the quasiatomic character of the localized ( here 5f) orbitals. Hence the localized electrons (5f) experience a spin- and orbital-dependent potential, while the other orbitals are delocalised and considered to be properly described by GGA. The rotationally invariant form of GGA+U is used with a spherically averaged double counting term [20]. Within this approximation, there is a single parameter $U_{\text {eff }}$ which is chosen to be equal to $4 \mathrm{eV}$ for all the studied compounds. Such a value is also very close to the value used by Dudarev et al. [13] or by Laskowski et al. [14] and to the experimental findings [21, 22].

A plane-wave basis set with a cutoff energy of $400 \mathrm{eV}$ was used in all calculations. Brillouin zone sampling was performed using a 16x16x16 k-point grid for the 12-atom primitive cell. This primitive cell allows studying the antiferrromagnetic ground state of $\mathrm{UO}_{2}$. The layered antiferromagnetic (AF) structure (see Fig.1) was assumed to be the one observed experimentally [2]. Such a k-point grid is found appropriate for total energies and magnetic moments to converge within $10^{-3} \mathrm{eV}$ and $0.01 \mu_{\mathrm{B}}$ respectively. Point defects were modelled using periodically repeated supercells consisting of $2 \times 2 \times 2$ primitive cells (96 atoms) with the number of $\mathrm{k}$ points reduced accordingly. Structural optimizations were performed at constant

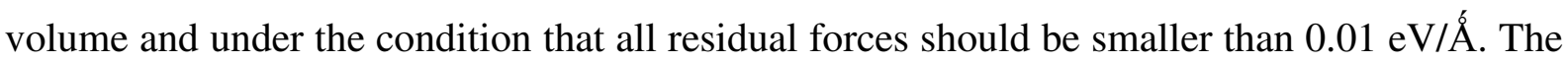
equilibrium properties of $\mathrm{UO}_{2}$ [cohesive energy, volume and density of states (DOS)] correspond to the ground-state configuration for the atomic and electronic degrees of freedom. 


\section{RESULTS AND DISCUSSION::}

\subsection{Pure compound $\mathrm{UO}_{2}$ :}

We first investigate the structural and thermodynamic properties of $\mathrm{UO}_{2}$ (fluorite structure, space group Fm3m). The equilibrium structure was determined by calculating the total energy for several different lattice parameters and fitting data to Birch-Murnaghan's equation of states. Three series of calculations were performed using different functionals, i.e. GGA, spin-polarized GGA (AF-GGA) and GGA+U. All the results are gathered in Table I. In contrast to previous results in the LDA [13], the GGA analysis shows that the antiferromagnetic structure is predicted to be the most stable structure. The calculated value of the magnetic moment localised on a uranium ion at equilibrium was found to be $1.44 \mu \mathrm{B}$, which is about $17 \%$ smaller than the value observed experimentally [2]. Compared to experiments, both GGA and AF-GGA give equilibrium lattice constants smaller than the experimental one while calculated cohesive energies are slightly larger than the experimental value. This latter overestimation is a well-known feature of GGA treatment. Consistent with prior research [9, 23], both GGA and AF-GGA give a metallic electronic structure, where the U $5 f$ bands are partially occupied (see Fig 2 a). In contrast, calculations using the GGA+U approach predict the correct insulating ground state with an energy band gap of $1.8 \mathrm{eV}$ in close agreement with the value observed experimentally (see Fig. 2b). Our analysis suggest that the insulating nature of the ground state results from taking better account of the effect of electron-electron correlations in the $5 \mathrm{f}$ band, in agreement with other theoretical analysis [13, 23]. Within the GGA+U approach, the magnetic moment of each $\mathrm{U}$ ion becomes $1.94 \mu \mathrm{B}$. Table I shows that the predicted lattice value constant is slightly larger than the experimental one while the calculated cohesive energy is smaller than the experimental one by about $6 \%$. All these results are consistent with weaker covalent bonding and suggest that the inclusion of correlation between $5 \mathrm{f}$ electrons indeed leads to this decrease binding. These findings may have also important implications for formation energies of point defects in $\mathrm{UO}_{2}$, characterized by the presence of strong correlations between valence electrons. 


\subsection{Point Defects:}

\section{- Isolated Point Defects:}

In the following, we consider neutral point defects, i.e. isolated vacancies and interstitials of uranium and oxygen. As already mentioned above, these point defects were modelled using periodically repeated supercells consisting of $2 \times 2 \times 2$ primitive cells (96 atoms). This corresponds to a defect concentration of $c_{U}=1 / 32$ and $c_{O}=1 / 64$. A specific supercell was built for each type of point defect. More particularly, the uranium interstitial has been chosen to be octahedrally coordinated. According to Willis [27], three different interstitial sites for oxygen have been considered: (i) the center of an oxygen cube $\mathrm{O}(0)$, (ii) the $\mathrm{O}(1)$ position is half way from the center of an oxygen cube and from the line joining two adjacent oxygen atoms. (iii) the $\mathrm{O}(2)$ position lies in the middle of the line joining the center of an oxygen cube and a summit of this cube.

The free energy of formation of an isolated point defect is defined as the free energy between the system with and without the defect:

$$
\Delta G_{f}=\Delta E_{f}-T \Delta S_{f}+P \Delta V_{f}
$$

Here $\Delta E_{f}$ is the change in total energy (including chemical potentials), $\Delta S_{f}$ is mainly the change in vibrational entropy, and $\Delta V_{f}$ is the change in volume effects when the defect is introduced into the system. As the defect concentration is small, the contribution of volume changes is small. When a defect is introduced, the associated change in the vibrational contribution has two distinct contributions: the defective lattice has a different number of vibrational modes compared with the perfect crystal; (ii) the frequencies of the remaining modes may change in the presence of the defect. However this contribution is known to be small and will be neglected. Then we focus only on computing formation energy terms. When investigating defects that change the composition of a material, the formation energies are usually evaluated with respect to a set of fixed external chemical potentials for the atomic species involved. The results will then depend on the actual values of these atomic chemical potentials. To be consistent with previous theoretical calculations, we chose for reference states the elements in their standard states, i.e. oxygen molecule and $\alpha$-uranium. Then free energies of formation for oxygen defects (oxygen interstitial: $I_{O}$ and oxygen vacancy: $V_{O}$ ) can be written as: 


$$
\begin{gathered}
\Delta E_{\left(\frac{V_{O}}{I_{O}}\right)}^{f}=E_{\left(\frac{V_{O}}{I_{O}}\right)}^{96 \mp 1}-E^{96} \pm \mu_{O} \\
\text { and } \mu_{0}=\frac{1}{2} \mu_{O_{2}}=\frac{1}{2}\left[E_{O_{2}}+k t \ln \left(f(T)+k t \ln \left(\frac{P_{0_{2}}}{P^{0}}\right)\right]\right.
\end{gathered}
$$

where the pressure reference state $\mathrm{P}^{0}=1 \mathrm{~atm}$, and $E_{\mathrm{O}_{2}}$ is the total energy of an isolated oxygen molecule that can be obtained from DFT calculations. Let us mention that the two last terms of equation (3) give a non negligible dependence of the free energy of formation of oxygen defects as a function of temperature and pressure. This dominating contribution to the free energy of formation then comes from the entropy increase associated with the release of gas molecules to the atmosphere.

The free energy of formation of uranium defects (uranium interstitial: $\mathrm{I}_{U}$ and uranium vacancy: $\left.\mathrm{V}_{\mathrm{U}}\right)$ are:

$$
\Delta E_{\left(\frac{V_{U}}{I_{U}}\right)}^{f}=E_{\left(\frac{V_{U}}{I_{U}}\right)}^{96 \mp 1}-E^{96} \pm \mu_{U}
$$

Let us mention that the $\mathrm{U}$ and $\mathrm{O}$ chemical potentials are not independent but must satisfy the equilibrium condition with $\mathrm{UO}_{2}$ bulk compound:

$$
\mu_{U}+\mu_{O}=F_{U O_{2}} \approx E_{U O_{2}}
$$

then the free energies of formation of the uranium defects depend also on both temperature and the oxygen pressure.

Table II gives the internal energies of formation of oxygen and uranium defects in $\mathrm{UO}_{2}$. They are also compared to previous ab initio based results.

We can see a qualitative agreement between the different sets of values. More particularly, for all the calculations, the formation energy of oxygen interstitials is predicted to be negative. This result means that $\mathrm{UO}_{2}$ compound is instable in presence of oxygen vapour, the incorporation of oxygen atoms occurring at the interstitial site of the fluorite structure. It is in agreement with the fact that $\mathrm{UO}_{2}$ spontaneously becomes oxidized in air at temperature as low as $120^{\circ} \mathrm{C}$ []. All other defects present positive formation energies. A quantitative comparison with the results of Petit et al. [] is difficult since their calculations are based on a very crude 


\section{- Defect interactions:}

We will finally present data and discuss the consequences of interactions between point defects in UO2. The most common approach to calculate the formation energies for more complex defects like Frenkel pairs, which consist of a vacancy and an interstitial of the same chemical type, and Schottky defects, which consist of two oxygen vacancies and one uranium vacancy, is to assume that vacancies and interstitials forming these defects are noninteracting. In this case, the formation energy of a Frenkel pair (FP) of $U$ (or O) specie is given by: 


$$
\Delta E_{F P(U, O)}^{f}=E_{V(U, O)}^{95}+E_{I(U, O)}^{97}-2 E^{96}
$$

and for the Schottky defect (S) by:

$$
\Delta E_{S}^{f}=E_{V(U)}^{95}+2 E_{V(O)}^{95}-3 \frac{95}{96} E^{96}
$$

The formation energies of these defects with the assumption of isolated point defects are reported in Table II. They are compared to previous theoretical results and also to values obtained by Matzke [6] from diffusion measurements. Let us mention that the experimental analysis of the populations of defects in $\mathrm{UO}_{2+\mathrm{x}}$ were performed using the point defect model (PDM), based on the assumption that the defects giving non-stoichiometry are isolated point defects. We can see that all theoretical results agree on predicting the larger stability of oxygen Frenkel pairs over uranium Frenkel pairs and Schottky defects. One can note also that the predicted formation energies of Frenkel pairs and Schottky defects are in the range of experimental data although calculations seem to overestimate the formation energy of the uranium Frenkel pair. However, let us mention that the experimental value of the uranium Frenkel pair given by Matzke [6] has been determined indirectly. Indeed it has been estimated from the measured formation energy of the oxygen Frenkel pair and the measured self diffusion activation energies of both oxygen and uranium by assuming that the oxygen point defects are dominant and point defects are isolated and non interacting.

To check the influence of interactions between point defects, we have calculated the formation energies of the uranium and oxygen Frenkel pairs using the LDA+U approach and the 96 atom supercell in which the vacancy and the interstitial forming the pair are either as far separated as possible (taking periodicity into account) or nearest neighbour. In the first case, the formation energies are equal to 10.2 and $2.8 \mathrm{eV}$ for uranium and oxygen Frenkel pairs respectively. These values are slightly less positive than those obtained from Equ. (6), indicating that the vacancy and the interstitial forming the Frenkel pair display weak attractive interactions. In the second case, when the two point defects are nearest neighbour, the findings are $0 \mathrm{eV}$ for both Frenkel pairs. These results show that the interstitial atom ( $\mathrm{U}$ or $\mathrm{O}$ atom) moves to the vacancy position without any barrier energy, emphasizing that such 


\section{SUMMARY AND CONCLUSIONS:}

In conclusion, we find that $\mathrm{DFT}+\mathrm{U}$ improves on the traditional density functionals, providing a first-principles description of $\mathrm{UO}_{2}$ in good agreement with experiment. This success is explained by the better treatment of the f-electron localization, which is responsible for the non zero band gap. Our calculations of formation energies of point defects show that this f-electron localization modify formation energies of uranium point defects more strongly than those of oxygen ones. Another important result is that the formation energies of Frenkel pairs and Schottky defects are very sensitive to defect interactions and then to the geometry of these defects. Therefore the assumption of non interacting defects commonly used in the literature must be considered very carefully. Further studies of more complex systems, such as "defect clusters" observed by experiments and suggested by our preliminary calculations as being energetically favourable, should be of interest. We believe that the results presented in this paper illustrate the usefulness of first-principles based calculations in elucidating complex structural behaviour in non-stoichiometric compounds.

\section{REFERENCES:}

[1] Schoenes J., 1980, Phys. Rep. 63, 301. 
[2] Faber J. Jr., Nader G.H., and Cooper B.R., 1975, Phys. Rev. Lett. 35,1770; Faber J. Jr., and Cooper B.R., 1976, Phys. Rev. B. 14,1151.

[3] Catlow C.R.A., 1977, Proc. R. Soc. London, Ser. A353, 533.

[4] Jackson R.A., Murray A.D., Harding J.H., and Catlow C.R.A, 1986, Phil. Mag. A53, 27.

[5] Jackson R.A., Catlow C.R.A. and Murray A.D., 1987, J. Chem. Soc., Faraday Trans. 2 83, 1171.

[6] Matzke H.J., 1973, J. Phys. Paris 34, C9-317; 1987, J. Chem. Soc. Faraday Trans 2, 83, 1121; 1990, ibid, 86, 1243.

[7] Petit T., Morel B., Lemaignan C., Pasturel A. and Bigot B., 1996, Phil. Mag. B 73, 893.

[8] Crocombette J.P., Jollet F., THien Nga L., and Petit T. 2001, Phys. Rev. B 64, 104107.

[9] Freyss M., Petit T., Crocombette J.P., 2005, J. Nucl. Mat., 347, 44.

[10] Kudin K.N., Scuseria G.E., and Martin R.L., 2002, Phys. Rev. Lett. 89, 266402.

[11] Prodan I.D., Scuseria G.E., and Martin R.L., 2006, Phys. Rev. B 73, 45104.

[12] Svane A., Temmerman W., Szotek Z., Laegsgard J. And Winter H., 2000, Int. J. Quantum Chem. 77, 799.

[13] Dudarev S.L., Nguyen Manh D., Sutton A.P., 1997, Phil. Mag. B 75, 613.

[14] Laskowski R., Madsen G.K.H., Blaha P., and Schwarz K., 2004, Phys. Rev. B 69, 140408(R).

[15] Kresse G. and Joubert D.,1999, Phys. Rev. B, 59, 1758.

[16] Kresse G. and Furthmüller J., 1996, Phys. Rev. B, 54, 11169; Kresse G. and Furthmüller J.,1996, Comput. Mat. Sci., 6, 15.

[17] Rohrbach A., Hafner J. and G. Kresse G., 2003, J. Phys.: Condens. Matter, 15, 979.

[18] Bengone O., Alouani M., Blöchl P.and Hugel J., 2000, Phys. Rev. B 62, 16392.

Rev. B, 49, 16223.

[19] Perdew J.P. and Wang Y., 1992, Phy. Rev. B 45, 13244.

[20] Dudarev S.L., Botton G.A., Savrasov S.Y., Humphreys C.J. and Sutton A.P., 1998, Phys. Rev. B, 57, 1505.

[21] Schoenes J., 1987, J. Chem. Soc., Faraday Trans. 2 83, 1205.

[22] Kotani A., and Yamazaki T., 1992, Progress Theor. Phys. Suppl. 108, 117.

[23] Yun Y., Kim Ha., Kim He., and Park K., 2005, Nucl. Eng. and Tech. 37, 293.

[24] Pascal P., 1967, Nouveau Traité de Chimie Minérale, T.15, Paris Masson

[25] Brooks M., and Kelly P., 1983, Solid St. Comm. 43, 793.

[26] Martin D.G., 1989, High Temp.-High Pressures 21, 13.

[27] Willis B.T.M., 1964, Proc. Br. Ceram. Soc. 1, 9. 
[28] Murray A.D., and Willis B.T.M, 1990, J. of Sol. State Chem. 84, 52. 
Figure Captions:

Figure 1: Fluorite crystal structure of $\mathrm{UO}_{2}$. Oxygen sites are in grey, uranium sites are shown in black. The antiferromagnetic ground state is characterized by the network of arrows.

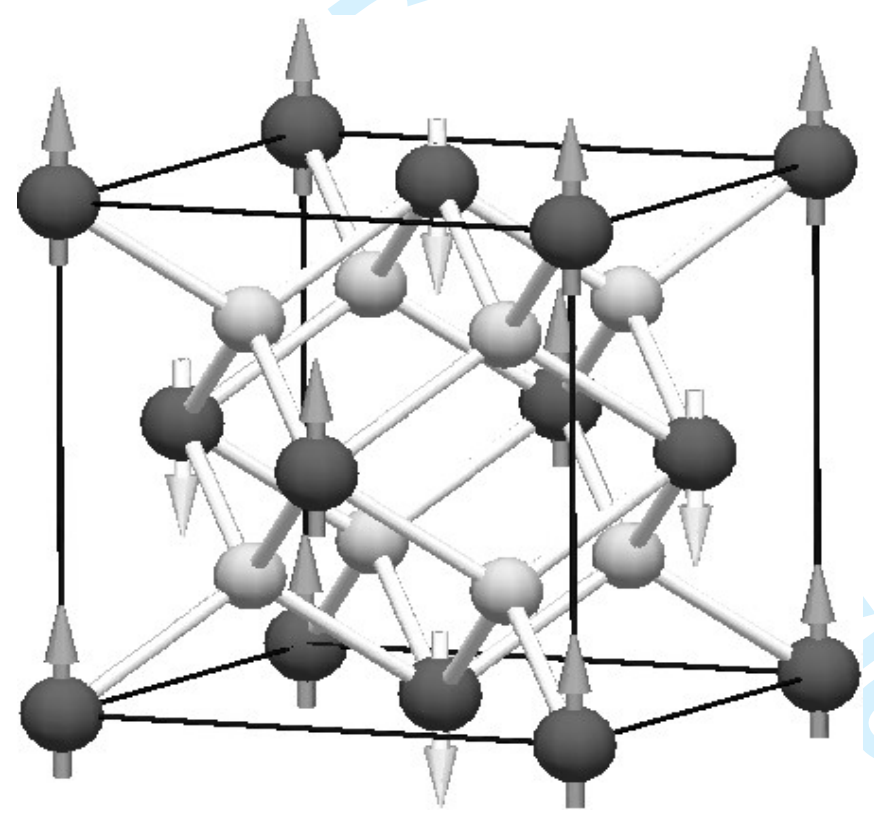


Figure 2: Total electronic DOS of $\mathrm{UO}_{2}$ : a) AF- GGA results. b) GGA+U.

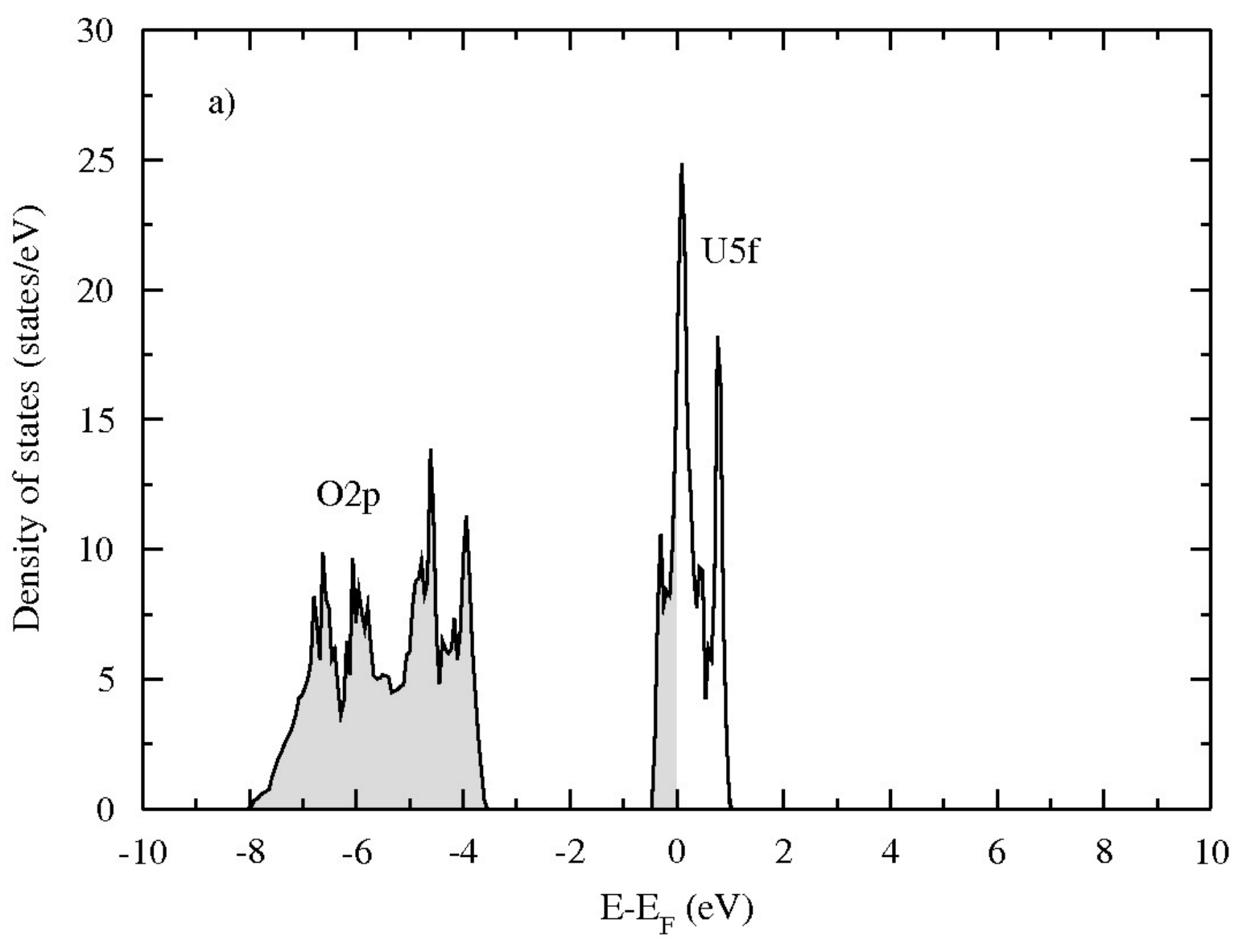




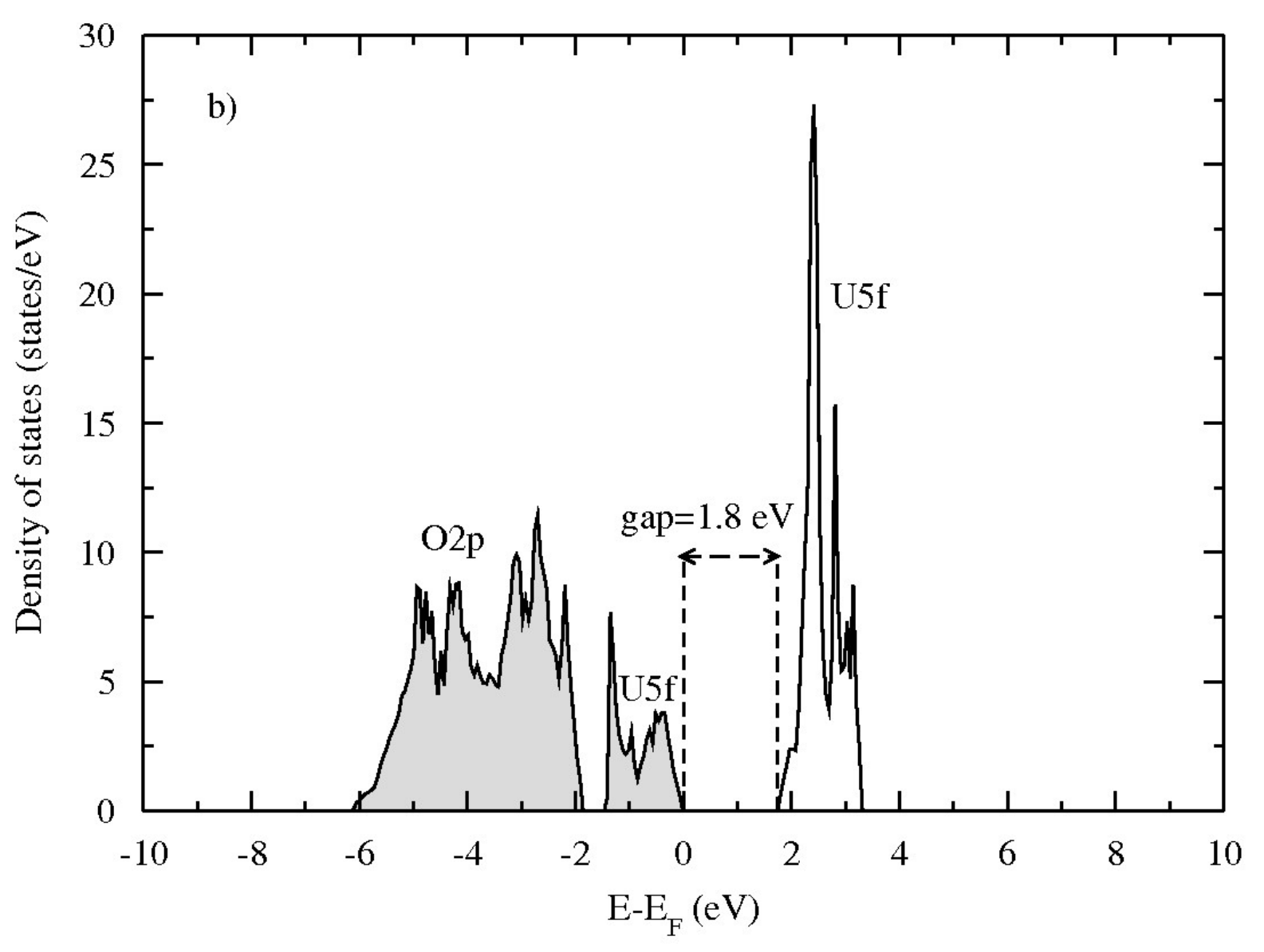



functionals i.e. GGA, AF-GGA and GGA+U.

\begin{tabular}{|l|l|l|l|l|}
\hline & GGA & AF-GGA & GGA+U & Experiment \\
\hline Lattice constant $(\mathrm{A})$ & 5.38 & 5.40 & 5.52 & $5.47[24]$ \\
\hline Cohesive energy $\left(\mathrm{eV} / \mathrm{UO}_{2}\right)$ & 23.58 & 23.62 & 21.71 & $22.31[25]$ \\
\hline Bulk modulus $(\mathrm{GPa})$ & 209 & 188 & 209 & $207[26]$ \\
\hline Magnetic Moment $(\mu \mathrm{B})$ & 0 & 1.44 & 1.94 & $1.74[2]$ \\
\hline Band gap $(\mathrm{eV})$ & 0 & 0 & 1.8 & $1.8[1]$ \\
\hline
\end{tabular}

Table I: The experimental and calculated properties of $\mathrm{UO}_{2}$ obtained from different 
Table II: Formation energies (eV) of point defects in UO2; uranium and oxygen vacancies (U-Vac and O-Vac), uranium and oxygen interstitials (U-Int and O-Int). Frenkel pairs (O-Fp and U-FP), and Schottky defect (S) are calculated as being non-interacting defects.

\begin{tabular}{|l|l|l|l|l|l|l|l|}
\hline Formation energy (eV) & U-Vac & O-Vac & U-Int & O-Int & $\begin{array}{l}\text { O-FP } \\
\text { (model) }\end{array}$ & $\begin{array}{l}\text { U-FP } \\
\text { (model) }\end{array}$ & $\begin{array}{c}\text { S } \\
\text { (model) }\end{array}$ \\
\hline GGA & 4.0 & 6.1 & 6.0 & -2.5 & 3.6 & 10.0 & 5.2 \\
\hline AF-GGA & 4.4 & 6.1 & 6.5 & -2.6 & 3.5 & 10.9 & 5.5 \\
\hline GGA+U & 6.0 & 5.6 & 8.2 & -1.6 & 4.0 & 14.2 & 7.2 \\
\hline LDA [7] & 19.1 & 10.0 & 11.5 & -3.3 & 6.7 & 30.6 & 17.1 \\
\hline LDA [8] & 3.3 & 6.7 & 7.3 & -2.9 & 3.8 & 10.6 & 5.8 \\
\hline GGA [9] & 4.8 & 6.1 & 7.0 & -2.5 & 3.6 & 11.8 & 5.6 \\
\hline Experimental [6] & & & & & $3.0-4.6$ & 9.5 & $6.0-7.0$ \\
\hline
\end{tabular}

